\title{
The treatment of nonunions with application of BMP-7 increases the expression pattern for angiogenic and inflammable cytokines: a matched pair analysis
}

\author{
This article was published in the following Dove Press journal: \\ Journal of Inflammation Research \\ 22 September 2016 \\ Number of times this article has been viewed
}

\author{
Patrick Haubruck' \\ Andreas Kammerer' \\ Sebastian Korff' \\ Philipp Apitz' \\ Kai Xiao ${ }^{2}$ \\ Axel Büchler' \\ Bahram Biglari ${ }^{3}$ \\ Gerald Zimmermann ${ }^{4}$ \\ Volker Daniel ${ }^{5}$ \\ Gerhard Schmidmaier ${ }^{1}$ \\ Arash Moghaddam' \\ 'Center for Orthopedics, Trauma \\ Surgery and Spinal Cord Injury, \\ Trauma and Reconstructive Surgery, \\ Heidelberg University Hospital, \\ HTRG - Heidelberg Trauma Research \\ Group, Heidelberg, Germany; \\ ${ }^{2}$ Department of Orthopedics \\ Wuhan, Wuhan Puai Hospital, \\ Tongji Medical College, Huazhong \\ University of Science and Technology, \\ Wuhan, People's Republic of China; \\ ${ }^{3}$ Department of Paraplegiology, \\ Berufsgenossenschaftliche Unfallklinik \\ Ludwigshafen, Ludwigshafen, \\ ${ }^{4}$ Department for Trauma Surgery, \\ Theresienkrankenhaus und St. \\ Hedwigs-Klinik GmbH, Mannheim, \\ ${ }^{5}$ Department of Transplantation \\ Immunology, Institute of Immunology, \\ University of Heidelberg, Heidelberg, \\ Germany
}

Correspondence: Patrick Haubruck

Center for Orthopedics, Trauma Surgery

and Spinal Cord Injury, Trauma and

Reconstructive Surgery, Heidelberg

University Hospital, HTRG - Heidelberg

Trauma Research Group, Schlierbacher

Landstrasse 200a, D-69I I 8 Heidelberg,

Germany

Tel +49622I 5635987

Email patrick.haubruck@med.uniheidelberg.de

\begin{abstract}
The local application of bone morphogenetic protein-7 (BMP-7) in combination with the transplantation of autologous bone graft improves the outcome in nonunion treatment; however, the specific reasons remain unclear. In this study, we sought to determine if the local application of BMP-7 contributes to improved bone regeneration in nonunion therapy by modulation of the angiogenic and inflammable cytokine expression patterns of the early inflammation response. Therefore, we utilized the analysis of serological cytokine expression patterns. As a matched pair analysis, best-fitting patients who were treated with transplantation of autologous bone graft (G1, $\mathrm{n}=10)$ were compared with patients who were treated with additional application of BMP-7 (G2, n=10). The changes in the cytokine expression patterns were monitored and correlated to clinical data of bone healing. Significant differences in angiogenesis potential (vascular endothelial growth factor [VEGF] serum levels) could be found in the first days after surgery $(P<0.05)$. Furthermore, the increase and absolute amount of VEGF levels in the BMP-7 group were considerably higher than in the control group during the first 2 weeks after surgery. The expression pattern of inflammable cytokines showed noticeable differences in the time point of significant elevated levels, in particular, inflammable cytokines showed an earlier peak in G2. Furthermore, interleukin-6 was significantly elevated within the first week only, comparing G2 to G1 $(P<0.05)$. Our findings indicate that BMP-7 induces an early and more intense expression of VEGF via a direct and postulated indirect pathway, thereby providing a favorable environment for bone healing. Moreover, application of BMP-7 leads to an earlier expression of known proinflammatory cytokines. The results of this study show that application of BMP-7 leads to costimulatory effect on both angiogenic and inflammable cytokine expression patterns that may serve as a possible stimulus for bone regeneration.
\end{abstract}

Keywords: bone regeneration, angiogenesis, VEGF, IL-6, inflammation, cytokines

\section{Introduction}

Despite modern treatment concepts, the therapy of nonunions remains a challenge in orthopedic and trauma surgery. According to the diamond concept, mechanical stability and biological factors such as angiogenesis, differentiated bone cells, and growth factors must be provided. ${ }^{1-9}$ Although the gold standard in nonunion therapy is the transplantation of autologous bone graft, missing bone regeneration subsequent to transplantation of autologous bone graft poses a serious disadvantage for the concerned patients. ${ }^{2,10-12}$ Therefore, in the last few years, this therapy was combined with the application of bone morphogenetic protein-7 (BMP-7) more and more frequently, 
and thereby, the use of BMP-7 in the current nonunion therapy was established. Studies show a better outcome after nonunion treatment if BMP-7 was applied..$^{713-15}$ The specific reasons still remain unresolved.

Previous studies reported local signs of inflammation after the application of BMP-7, such as swelling or soft-tissue inflammation ${ }^{13,16}$ without signs of systemic inflammation; in particular, no persistent increase in serological infectious parameters (such as C-reactive protein [CRP] and leukocytes) has been shown. However, patients and caregivers remain disconcerted by this local infectious reaction. ${ }^{14}$ The inflammatory response marks the start of the fracture healing cascade. ${ }^{17-19}$ Important inflammatory mediators are the vascular endothelial growth factor (VEGF), tumor necrosis factor- $\alpha$ (TNF- $\alpha$ ), and several interleukins (ILs), such as interleukin-6 (IL-6) and interleukin-8 (IL-8). The serum levels of the proinflammatory cytokines, such as IL-6 and TNF- $\alpha$, initially increase within the first 24 hours after trauma. ${ }^{12}$ These cytokines play a key role in the inflammatory response. ${ }^{20} \mathrm{VEGF}$ is of particular interest for its impact on not only inflammation but also angiogenesis. ${ }^{21}$ However, the effect of BMP-7 on the early inflammatory response has hardly been explored and is the subject of the current research.

In previous studies, we could show that the serum analysis of the cytokine expression pattern is a validate tool in investigating the underlying principles of bone regeneration. ${ }^{22,23}$ Hence, in this study, we sought to improve the understanding of the modulating effect of the adjunct application of BMP-7 on the angiogenic and inflammable cytokine expression patterns, in particular the early inflammatory response by utilizing this established protocol.

\section{Patients and methods}

\section{Study design}

Between 2009 and 2014, 109 patients of the Berufsgenossenschaftliche Unfallklinik Ludwigshafen (BG Trauma Centre) and the Center for Orthopaedics and Trauma Surgery of the University Hospital Heidelberg were enrolled in this prospective clinical observer study, subsequent to a written declaration of consent given by all of them. In the current study, the cytokine serum levels of VEGF, TNF- $\alpha$, and other ILs were compared between two groups: patients who were treated with a transplantation of autologous bone graft (G1) and patients who were treated with an additional application of BMP-7 (G2). The changes in the cytokine expression patterns were monitored during the preoperative examination and additionally at standardized intervals after surgery. Furthermore, all enrolled patients were invited to attend follow-up examinations both clinically and radiologically. The patients included in our study were examined radiologically and clinically at the following time points: 1, 2, 4, and 6 weeks as well as 3, 6, and 12 months after surgery; 6 months after surgery, the patients could be declared responders or nonresponders due to radiological signs of consolidation and clinical signs of mechanical stability and full weight bearing. The serum levels of CRP, leukocytes, VEGF, TNF- $\alpha$, IL-1, IL-6, IL-8, and IL-10 were analyzed and correlated to clinical data of bone healing. The study was conducted in accordance with the Declaration of Helsinki, and it was approved by the ethics committee of the Ruprechts-Karls-Universität Heidelberg (S-636/2011 and 157/2002).

\section{Patient characteristics and surgical procedure}

In this clinical observer study, two groups of patients were compared. In total, we acquired blood samples of 59 patients from Heidelberg and 50 patients from Ludwigshafen with one-step procedure after nonunion. Out of the 109 patients, BMP-7 was used in 33 patients. Eleven of these patients did not show up consistency for follow-up examination as well as blood sample collection and had to be eliminated from the study. One patient had a nonunion close to the ankle joint and three patients did not heal in time and thus were also excluded from this study, leaving 18 patients with the matching criteria. Of the 76 patients without the application of BMP-7, 25 had a nonunion in a bone other than tibia or femur. Eighteen patients either got a subsequent operative procedure or were treated aberrantly to the sole application of autologous bone graft. Of 22 patients, we had less blood samples than needed. This left a remaining eleven patients fitting this study's criteria. From these patients, we were able to form a group including five men and five women who were treated with solely the transplantation of autologous cancellous bone graft, serving as control (G1). As a matched pair analysis, we took the bestfitting five men and five women out of the patients fitting criteria, who were treated with a transplantation of autologous cancellous bone graft and with an additional application of BMP-7 to form the study group (G2; Table 1).

\section{G2 group in detail}

Five female and 5 male patients were assigned to this group. The mean age at the time of surgery was $51.5 \pm 9.91$ years. The mean body mass index (BMI) was $30.59 \pm 6.91$ with a range of 21.48-43.21. Five patients suffered from a femoral nonunion, and the others had a tibial nonunion. Four patients were smoker, one patient was a former smoker (within the last 10 years), 
Table I Patient's characteristics and demographics comparing the BMP-7 group to the control group

\begin{tabular}{|c|c|c|c|c|c|c|c|c|c|c|c|c|c|c|}
\hline \multirow[t]{2}{*}{ Patients } & \multirow[t]{2}{*}{$\mathbf{n}$} & \multicolumn{2}{|l|}{ Sex } & \multirow[t]{2}{*}{ Age (years) } & \multirow[t]{2}{*}{ BMI $\left(\mathbf{k g} / \mathbf{m}^{2}\right)$} & \multicolumn{3}{|c|}{ Smoking } & \multicolumn{2}{|c|}{ Diabetes } & \multicolumn{2}{|c|}{ Localization } & \multicolumn{2}{|c|}{ Fixation } \\
\hline & & Female & Male & & & $\mathbf{S}$ & FS & NS & Yes & No & Tibia & Femur & Nail & Plate \\
\hline All patients & 20 & 10 & 10 & $52.00 \pm 9.73$ & $28.86 \pm 6.45^{\mathrm{a}}$ & 7 & 4 & 9 & 0 & 20 & 8 & 12 & 9 & II \\
\hline Control group (GI) & 10 & 5 & 5 & $52.50 \pm 10.1$ & $26.94 \pm 5.65^{b}$ & 3 & 3 & 4 & 0 & 10 & 3 & 7 & 1 & 9 \\
\hline Intervention group (G2) & 10 & 5 & 5 & $51.50 \pm 9.9$ & $30.59 \pm 6.91$ & 4 & 1 & 5 & 0 & 10 & 5 & 5 & 8 & 2 \\
\hline
\end{tabular}

Notes: Age and BMI data presented as mean \pm SD. Smoking is divided into three subgroups (S, FS, and NS). alndicates data from only 19 patients. ${ }^{b}$ Indicates data from only 9 patients.

Abbreviations: BMP-7, bone morphogenetic protein-7; BMI, body mass index; SD, standard deviation; S, smoker, FS, former smoker, NS, nonsmoker; GI, patients who were treated with transplantation of autologous bone graft; G2, patients who were treated with additional application of BMP-7.

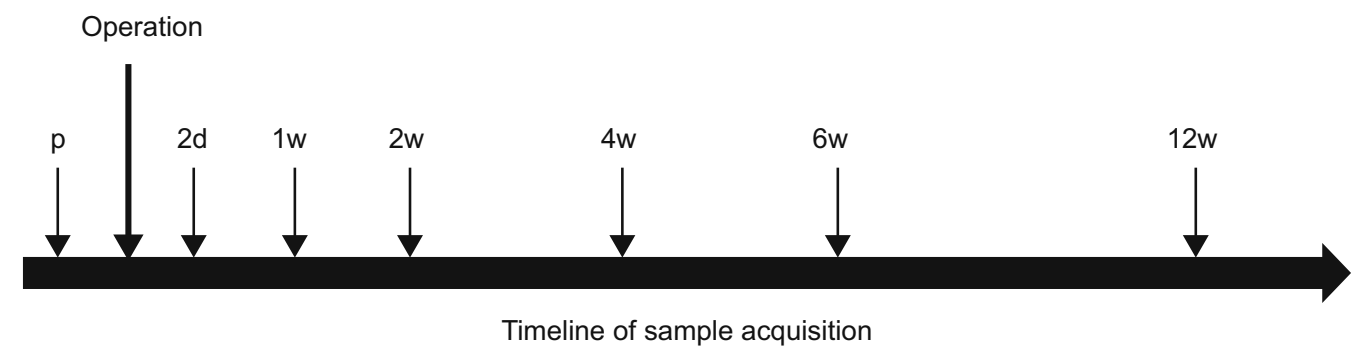

Figure I A timeline of the protocol for taking blood samples throughout this study.

Notes: Blood was drained from each patient Id $p$ and $2 d, I w, 2 w, 4 w, 6 w$, and $12 w$ after surgery.

Abbreviations: p, preoperative; d, day(s); w, week(s).

and five patients were nonsmokers. ${ }^{24}$ Arterial hypertension was diagnosed in five patients. Nonunion fixation was done by nailing in eight cases and by plate in two cases (Table 1).

\section{GI group in detail}

The control group also consisted of five female and five male patients. The mean age was $52.5 \pm 10.05$ years. The mean BMI was $26.94 \pm 5.65$ with a range of 21.55-39.45. The BMI of one patient could not be determined; thus, the mean BMI was calculated from the data of the remaining patients $(n=9)$. There were five tibial and five femoral nonunions. Three patients were smokers, three were former smokers (within the last 10 years), and four were nonsmokers. ${ }^{24}$ Four patients suffered from arterial hypertension. Plate fixation was used in nine cases and fixation by nailing in one case (Table 1).

Herewith, both groups were comparable in sex, age, BMI, location of fracture, smoking habits, and diseases. Furthermore, for each patient, the surgical treatment involved open reduction and internal fixation with fixation either by nailing or plate fixation.

\section{Sample acquisition and analysis of serum cytokine levels}

First blood samples were taken during the preoperative examination. Additional samples were taken postoperatively at standardized intervals, on days 2 and 7 and on 1, 2, 4, 6, and 12 weeks after surgery (Figure 1). The samples were collected between 8 am and $11 \mathrm{am}$. In all, $3 \times 7.5 \mathrm{~mL}$ serum samples (S-Monovette $7.5 \mathrm{~mL}$; Sarstedt AG \& Co., Nümbrecht, Germany) and $1 \times 9 \mathrm{~mL}$ ethylenediaminetetraacetic acid were taken, centrifuged, aliquoted into Eppendorf tubes and stored at $-80^{\circ} \mathrm{C}$. Furthermore, $\mathrm{C}$ reactive protein (CRP) and leukocyte serum values were assessed during our clinical routine in the first 2 weeks to analyze systemic inflammation. Herewith, we assessed if application of BMP-7 and autologous cancellous bone graft influences systemic CRP and leukocyte levels and thereby a systemic unspecific inflammation that might modulate the cytokine serum values. Furthermore, a possible bias by infection or postoperative ongoing elevated CRP and leukocyte blood levels could be detected. Luminex Performance Human High Sensitivity Assays (Quantikine ${ }^{\circledR}$; R\&D Systems, Inc., Minneapolis, MN, USA) were used to measure the cytokine serum levels of VEGF, TNF- $\alpha$, IL-1, IL-6, IL-8, and IL-10 as described in the factory manual. The lab technician performing the Luminex assays was blinded to patient data and kept to manufacturer's instructions during the measurements.

\section{Statistical analysis}

The serum levels were expressed as absolute mean concentrations \pm standard deviation. To show significant differences between time points in one study group, the nonparametric Wilcoxon signed rank test was used. The Mann-Whitney $U$ test was used to show significant differences between 
both study groups. Statistical significance was determined as $P=0.05$. Statistical analyses were carried out with the SPSS Statistics version 2.0 (SPSS Inc., Chicago, IL, USA). The graphs were created with the SigmaPlot software (Systat Software Inc., San Jose, CA, USA).

\section{Results}

In this prospective clinical observer study, we examined patients with long bone fractures that were treated with transplantation of autologous cancellous bone graft $(\mathrm{G} 1)$ and adjunct local application of BMP-7 (G2) and compared their specific time course of cytokine expression (VEGF, TNF- $\alpha$, IL-1, IL-6, IL-8, and IL-10) to their control group.

\section{Differences in VEGF serum-level pattern}

Significant differences in angiogenesis potential (VEGF serum levels) could be found in the first days after surgery comparing G2 and the control groups. In detail, the VEGF serum levels in the BMP-7 group rose from day 2 after surgery to a peak on day 14 and were finally comparable to those at admission 4 weeks after surgery (Figure 2A). In contrast, the VEGF serum levels in our control group decreased after surgery on day 2 after surgery and afterward showed a VEGF time course that was comparable to the G2 group, but in contrast to this, the absolute serum levels of VEGF showed significant differences before surgical treatment $(P=0.035)$ and on day 2 after surgery $(P=0.009)$ comparing the study group and control group. Furthermore, the absolute increase in the VEGF serum levels between preoperative values and values at week 2 was considerably higher in the G2 group compared to the control group (absolute increase 2 weeks vs preoperative: G2, $186.60 \mathrm{pg} / \mathrm{mL}$ and $\mathrm{G} 1,100.53 \mathrm{pg} / \mathrm{mL}$ ). In summary, the increase (Figure 2B) and absolute amount of VEGF levels in the BMP-7 group were considerably higher than in the control group during the first 2 weeks after surgery (G2 after 2 weeks: 351.95 pg/mL; G1 after 2 weeks: $191.57 \mathrm{pg} / \mathrm{mL}$ ) as shown in Figure 2.

\section{Differences in TNF- $\alpha$ serum-level pattern}

TNF- $\alpha$ was similarly elevated after surgery in both groups (control and study). The mean concentrations of TNF- $\alpha$ in the BMP-7 group peaked 1 week after treatment and returned to preoperative serum levels of $12.0 \pm 3.3 \mathrm{pg} / \mathrm{mL}$ after 6 weeks (Figure 3A). In contrast to the study group, an initial decrease in the TNF- $\alpha$ serum level was observed in the control group 2 days after surgery. Interestingly, the maximum TNF- $\alpha$ concentration was measured not merely at week 1 but rather 4 weeks after surgery $(17.2 \pm 2.8 \mathrm{pg} / \mathrm{mL})$. No significant differences could be found in comparing both groups at each time point. However, noticeable differences could be detected in the time point of significant elevated TNF- $\alpha$ levels. In the G2 group, peak values of TNF- $\alpha$ serum

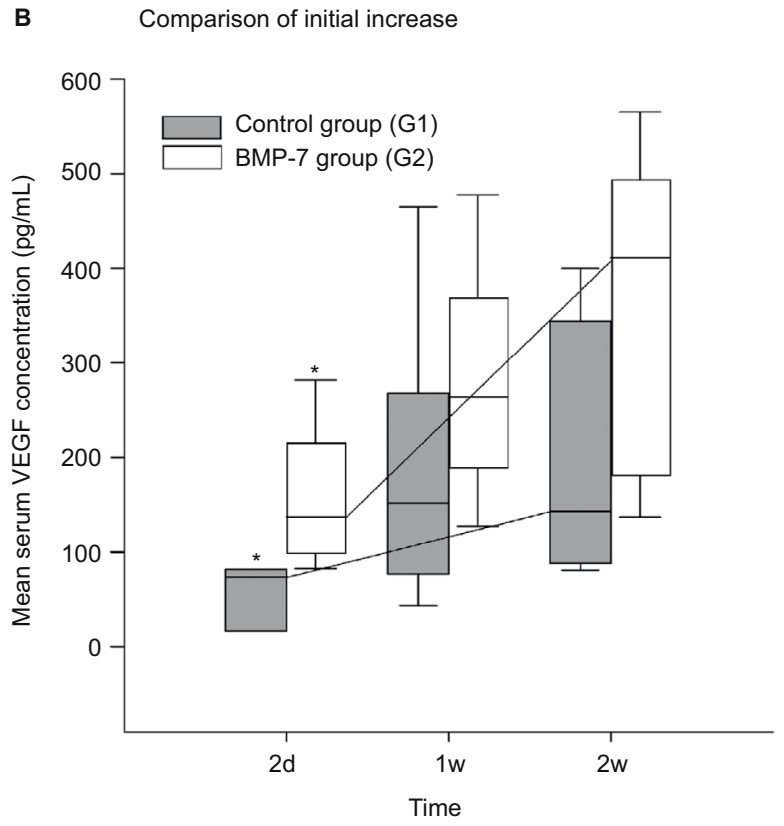

Figure 2 Evaluation of the expression pattern of VEGF after nonunion treatment. Notes: (A) Serum concentrations of VEGF in pg/mL Id before and $2 \mathrm{~d}, \mathrm{Iw}, 2 \mathrm{w}, 4 \mathrm{w}, 6 \mathrm{w}$, and I $2 \mathrm{w}$ after surgery. Significant differences are indicated by a star $(P<0.05$, whiskers are SD). (B) Box plot after $2 \mathrm{~d}$ to $2 \mathrm{w}$ of surgery. Whiskers demonstrate one-quarter of the sample. Stars indicate significant differences comparing the study and control groups at the time point $(P<0.05)$.

Abbreviations: VEGF, vascular endothelial growth factor; p, preoperative; d, day(s); w, week(s); SD, standard deviation; BMP-7, bone morphogenetic protein-7; GI, patients who were treated with transplantation of autologous bone graft; G2, patients who were treated with additional application of BMP-7. 

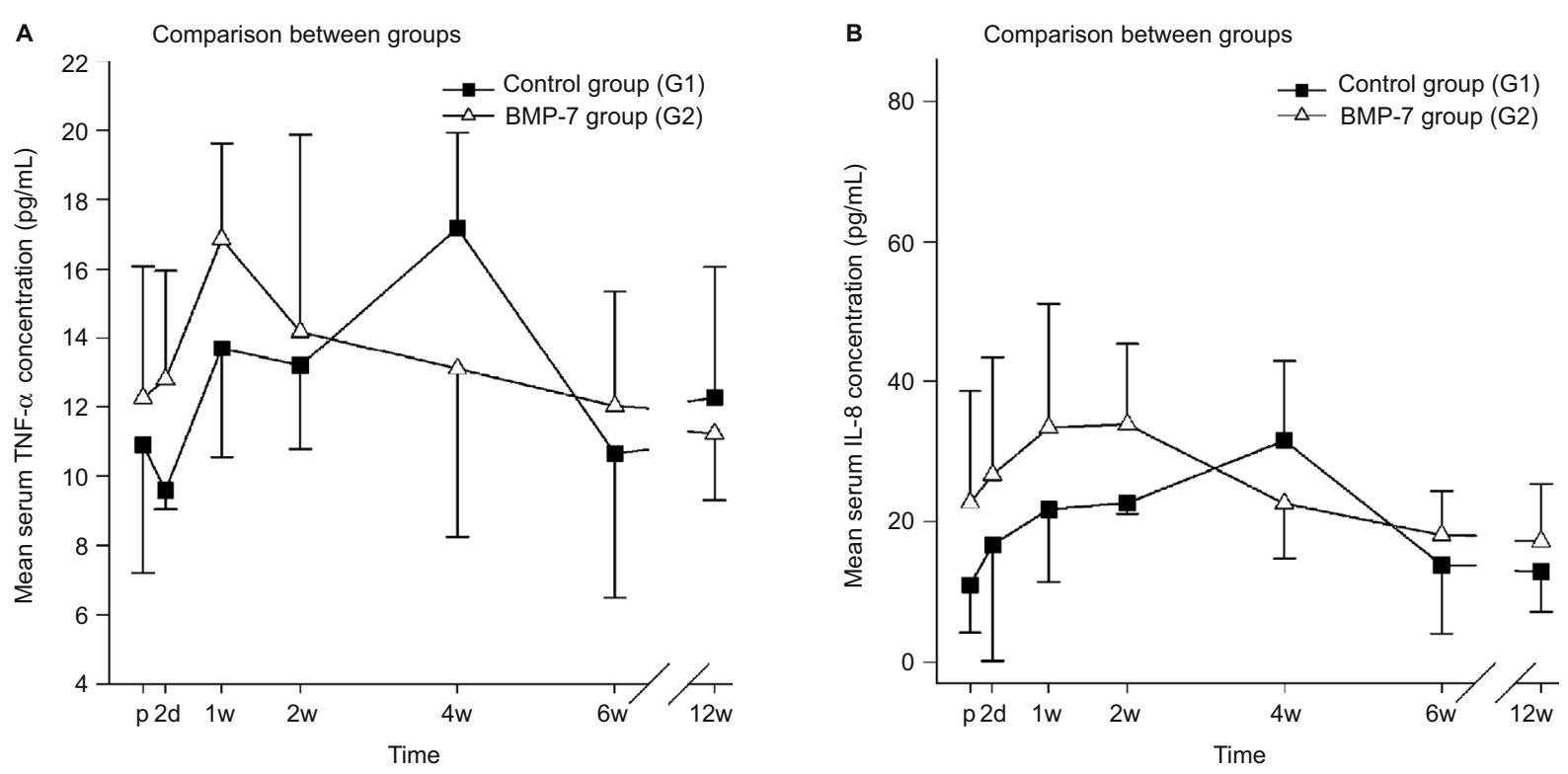

Figure 3 Evaluation of the expression pattern of TNF- $\alpha$ and IL-8 after nonunion treatment.

Notes: (A) Serum concentrations of TNF- $\alpha$ in pg/mL Id before and $2 \mathrm{~d}, \mathrm{Iw}, 2 \mathrm{w}, 4 \mathrm{w}, 6 \mathrm{w}$, and I $2 \mathrm{w}$ after surgery. (B) Serum concentrations of IL-8 in pg/mL Id before and $2 \mathrm{~d}, \mathrm{Iw}, 2 \mathrm{w}, 4 \mathrm{w}, 6 \mathrm{w}$, and I2w after surgery. Peak of IL-8 in the control group could be found after $4 \mathrm{w}$ of surgery. Peak of IL- 8 in the study group was found within the first $2 w$ after surgery.

Abbreviations: TNF- $\alpha$, tumor necrosis factor- $\alpha$; p, preoperative; d, day(s); w, week(s); SD, standard deviation; IL-8, interleukin-8; BMP-7, bone morphogenetic protein-7; $\mathrm{GI}$, patients who were treated with transplantation of autologous bone graft; G2, patients who were treated with additional application of BMP-7.

levels could be detected on week 1 comparing to peak values of TNF- $\alpha$ serum levels in the G1 group at week 4 (Figure 3A). These data imply an earlier peak in the TNF- $\alpha$ plasma levels of the BMP-7 group than those of the control group by more than 2 weeks.

\section{Differences in IL-8 serum-level pattern}

IL-8 did not show any significant differences comparing both groups at each measured time point. However interestingly, the highest values in both groups could be found at different time points during the measured time course after surgery with an earlier peak in the IL- 8 serum levels of the BMP-7 group. In detail, the BMP-7 group showed IL-8 peak serum values after 2 weeks and a decrease in values near to admission after 4 weeks (22.6 $\pm 7.8 \mathrm{pg} / \mathrm{mL}$; Figure 3B). Compared to this, the levels of admission were lower in the control group, followed by a constant increase until a peak value at week 4 . The decrease to levels of admission was reached 6 weeks after surgery $(13.9 \pm 9.8 \mathrm{pg} / \mathrm{mL})$. No statistical significances could be found comparing both groups at each time point, but an earlier peak was observed in the BMP-7 group (week 2) compared to the control group (week 4; Figure 3B).

\section{Differences in IL-6 serum-level pattern}

IL-6 was significantly elevated within the first week only, comparing G2 group to the G1 group. In detail, the IL-6 levels in the BMP-7 group increased to a peak mean concentration 2 days after surgery. Afterward, IL-6 plasma values decreased to similar levels at admission $(4.9 \pm 1.0 \mathrm{pg} / \mathrm{mL})$ within the first 2 weeks after surgical therapy (Figure 4A). Compared to this, in the control group, the levels of IL- 6 at admission were similar to those in the G2 group. Furthermore, the IL-6 serum levels in the control group after 1 week of surgery were similar $(6.1 \pm 3.0 \mathrm{pg} / \mathrm{mL})$ to those at admission or to those in the BMP-7 group after 2 weeks of surgery. However in contrast, the peak value on day 2 was significantly lower $(9.1 \pm 3.0 \mathrm{pg} / \mathrm{mL})$ in the control group than any other value in the BMP-7 group within the first week after surgery. Additionally, during the following time course, a constant decrease was observed within a minimum 6 weeks after surgery $(1.6 \pm 0.8 \mathrm{pg} / \mathrm{mL}, P=0.014$ compared to the BMP-7 group). Additionally, the absolute increase on day 2 compared to the admission level was higher in the BMP-7 group (absolute increase: $59.2 \mathrm{pg} / \mathrm{mL}$ ) than in the control group (absolute increase: $7.1 \mathrm{pg} / \mathrm{mL}$; Figure 4B). This increase and peak value is significant on day 2 comparing both study groups $(P=0.049)$, suggesting an increased process of inflammation in the BMP-7 group.

\section{Pattern of IL-I and IL- I 0 serum levels}

In this study, we thoroughly assessed the influence of BMP-7 application on the IL serum pattern. However, no measurable values of IL-1 and IL-10 could be found using plasma samples as described earlier. 
A

Comparison between groups

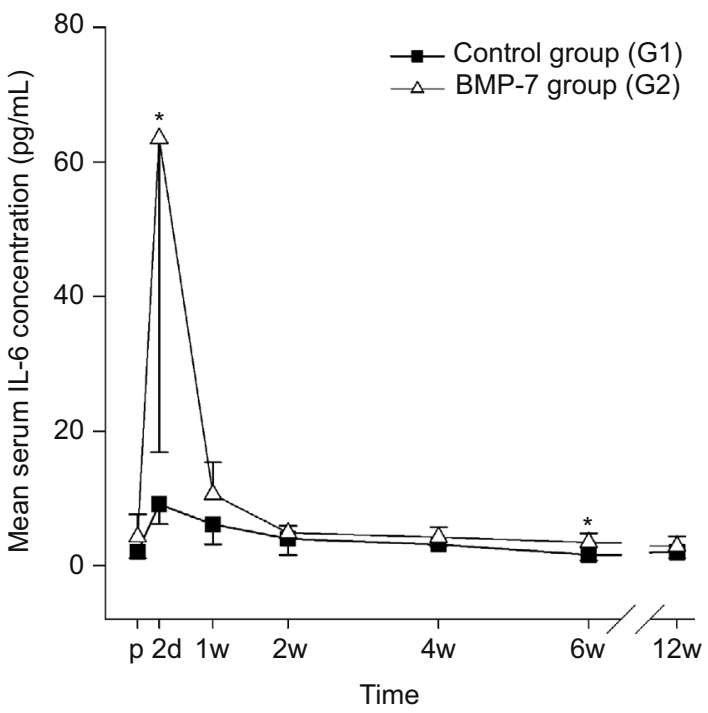

B Highlight on the initial time course

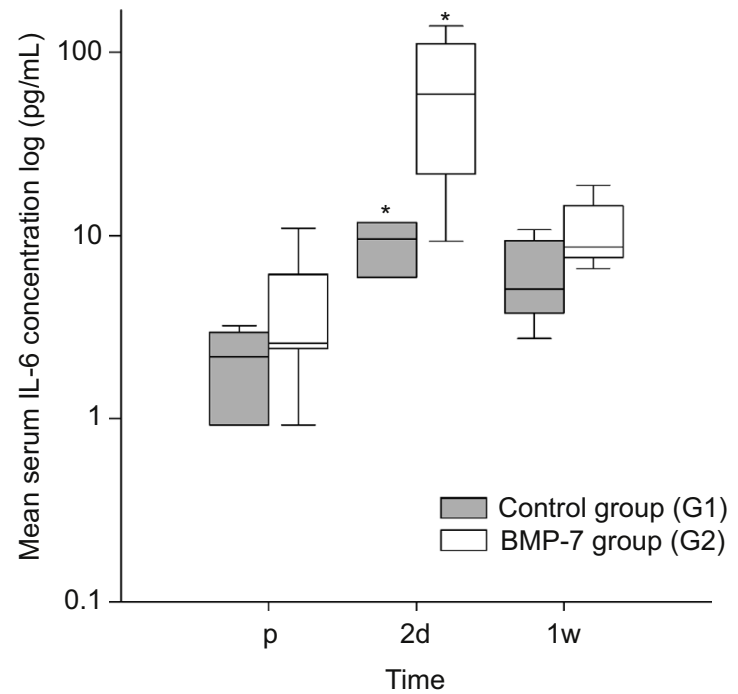

Figure 4 Evaluation of the expression pattern of IL- 6 after nonunion treatment.

Notes: (A) Serum concentrations of IL- 6 in pg/mL Id before and $2 d, I w, 2 w, 4 w, 6 w$, and I $2 w$ after surgery. Significant differences are indicated by a star $(P<0.05$, whiskers are SEM). (B) Box plot in a logarithmic scale on the $y$-axis of day before to I w after surgery. Whiskers demonstrate one-quarter of the sample. Stars indicate significant differences comparing the study and control group at the time point $(P<0.05)$.

Abbreviations: IL-6, interleukin-6; p, preoperative; d, day(s); w, week(s); SEM, standard error of the mean; BMP-7, bone morphogenetic protein-7; GI, patients who were treated with transplantation of autologous bone graft; G2, patients who were treated with additional application of BMP-7.
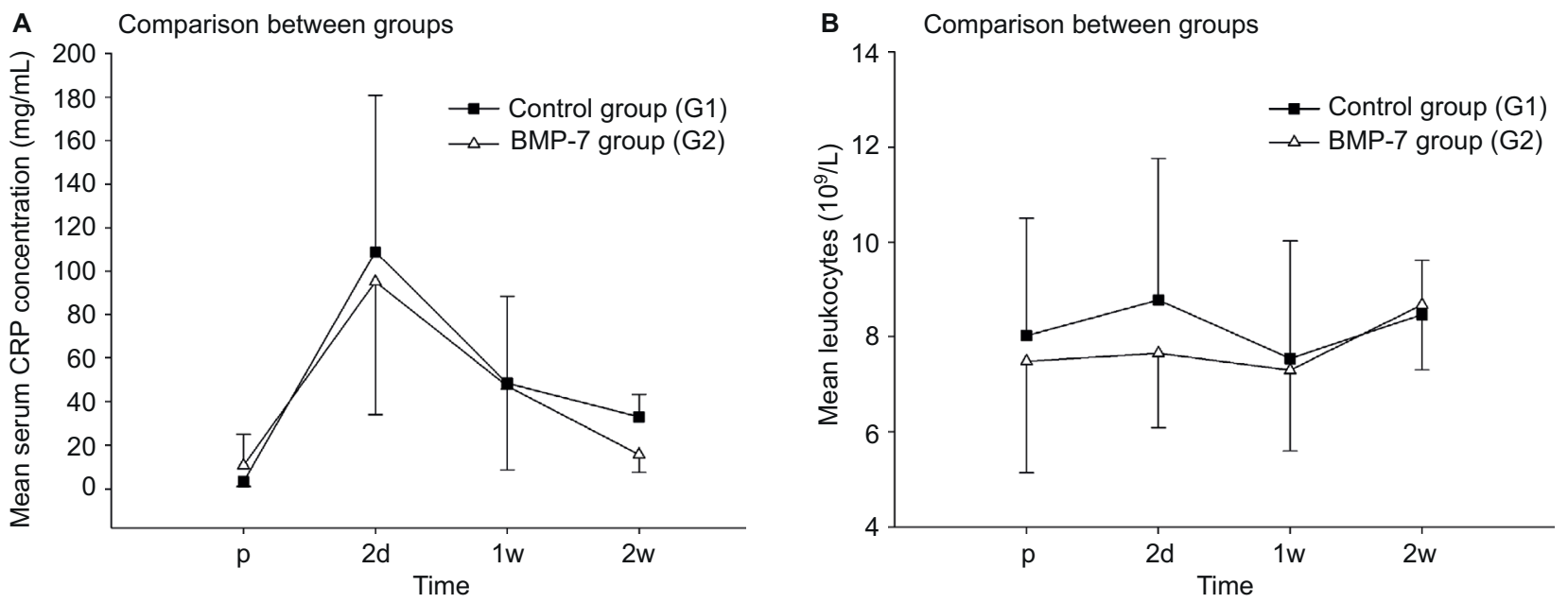

Figure 5 Analysis of the serological inflammable parameters after nonunion treatment.

Notes: (A) Serum concentrations of CRP in $\mathrm{mg} / \mathrm{L}$ Id before and $2 \mathrm{~d}$, I w, and $2 \mathrm{w}$ after surgery. (B) Serum concentrations of leukocytes in I,000/nL Id before and $2 \mathrm{~d}$, I w, and $2 \mathrm{w}$ after surgery.

Abbreviations: CRP, C-reactive protein; p, preoperative; d, day(s); w, week(s); BMP-7, bone morphogenetic protein-7; GI, patients who were treated with transplantation of autologous bone graft; G2, patients who were treated with additional application of BMP-7.

\section{Differences in CRP and leukocyte serum- level patterns}

During our follow-up, we assessed CRP and leukocytes in the initial 2 weeks only. As expected, preoperatively, both groups showed similar values (study group $10.72 \pm 14.248 \mathrm{mg} / \mathrm{L}$ compared to control group 3.19 $\pm 2.094 \mathrm{mg} / \mathrm{L}$ ), and subsequent to surgery, both groups showed an expected increase that showed similar peak values at the second day after surgery (study group $95.02 \pm 60.996 \mathrm{mg} / \mathrm{L}$ compared to control group 108.86 $\pm 71.908 \mathrm{mg} / \mathrm{L}$; Figure 5A). Interestingly, a decrease in the serum values of CRP after 2 weeks of surgery was significant in the control group (study group $15.67 \pm 8.167 \mathrm{mg} / \mathrm{L}$ compared to control group $33.07 \pm 10.221 \mathrm{mg} / \mathrm{L}, P=0.037$ ). The serum values of leukocytes showed a similar pattern during the complete study period, but values of both groups stayed in the physiological range (range 4,500-13,000/nL; Figure 5B). 


\section{Discussion}

In this clinical observer study, we analyzed the serum concentrations of VEGF, TNF- $\alpha$, IL- 6 , and IL- 8 after nonunion treatment with the transplantation of autologous bone graft (control group) and adjunct application of BMP-7 (control group). A better bone healing after the additional application of BMP-7 to the standard nonunion therapy has already been characterized. ${ }^{7,13,14,25}$ We sought to determine if the adjunct application of BMP-7 modulates the cytokine expression pattern of angiogenic and inflammable cytokines, in particular the early inflammatory response. After the application of BMP-7, an earlier expression of all measured cytokines was observed, but only a higher peak value of VEGF and IL-6 was detected after the application of BMP-7 compared to the matched control group. The strengths of this study are its prospective approach, the clear inclusion and exclusion criteria, and the matching of patients. This allowed us to eliminate factors that could bias the evaluation of these patient groups.

\section{Angiogenesis}

Large defect sizes present great challenges in nonunion therapy. Therapy options are the transplantation of allo- and autografts or implants. The transplanted material has no chance of survival or integration into the fracture gap if no adequate vascular supply is given. ${ }^{26} \mathrm{~A}$ sufficient angiogenesis is needed shortly after fracture to secure the following bone regeneration. ${ }^{27}$ The expression of VEGF promotes angiogenesis; in particular, angiogenesis is the formation of new blood vessels in a regulated mechanism that is still not completely characterized. It is known that a total inhibition of angiogenesis causes a failure of fracture healing. ${ }^{28}$ The most important mediator of angiogenesis is VEGF. ${ }^{29}$ VEGF induces the inflammatory response and contributes to the transformation of soft-to-hard callus. ${ }^{30}$ Analyses of VEGF levels in the human serum samples showed an increase in the VEGF levels up to 2 weeks after fracture. ${ }^{31}$ These findings were confirmed by our results, but furthermore, our results did show a tendency for higher VEGF plasma levels in the study group. In our study, the VEGF levels in the BMP-7 group were elevated prior to local application of BMP-7 and the expression pattern of VEGF was similar in both groups. However, subsequent to adjunct application of BMP-7, VEGF levels showed both a steeper and faster increase in the first weeks of nonunion treatment and a higher absolute increase compared to the baseline value. Thereby, our data indicate a modulation of the VEGF expression after the application of BMP-7 that might be favorable for angiogenesis. In this study, we were able to postulate two different pathways that might achieve this modulation. Further studies are needed to confirm our postulations.

\section{Direct stimulation of the VEGF expression by BMP-7}

In recent years, multiple studies found a considerable role played by VEGF next to BMPs in osteogenesis. Therefore, a number of studies investigated the relationship of VEGF and BMP and found synergistic actions of VEGF and BMP-2 on bone healing. Furthermore, they could show that BMPs increased VEGF expression in osteoblasts. ${ }^{32}$ Animal studies showed better bone regeneration after nonunion therapy with additional VEGF application. ${ }^{33-35}$ Additionally, a stimulation of angiogenesis via BMP-signaling was demonstrated in in vitro studies. ${ }^{36-38}$ Akiyama et al ${ }^{39}$ pointed out that BMP-7 induces VEGF expression in granulosa cells as well as umbilical cord endothelium. Thereby, the results of our study indicate that the local application of BMP-7 increases the serum levels of VEGF by direct stimulation of the VEGF expression during nonunion therapy. The local application of BMP-7 let to a longer and higher VEGF expression.

\section{Indirect stimulation of the VEGF expression by costimulatory effects of inflammable cytokines}

Angiogenesis is a complex and multistep process that is regulated by many different growth factors and cytokines (eg, platelet-derived growth factor and basic fibroblast growth factor). VEGF is a very potent angiogenic agent that acts as a specific mitogen for endothelial cells through specific cell surface receptors. However, the molecular mechanism regulating the expression of VEGF is not characterized, and it is likely that other cytokines might promote the expression of VEGF as well. Cohen et al reported that the induction of IL-6 promotes the expression of VEGF during hypoxia, wound healing, and ovulation and should be considered as an indirect angiogenic factor. Furthermore, they reported that TNF- $\alpha$ has been implicated as an indirect angiogenic factor most probably by induction of the expression of VEGF. ${ }^{40,41} \mathrm{In}$ other studies, IL- 8 has been shown to be a promoting factor for angiogenesis and to interact with VEGF., ${ }^{42,43}$ The results of our study showed an earlier peak of IL- 8 and TNF- $\alpha$ correlating with the steeper initial increase in the VEGF expression pattern. Furthermore, the IL-6 expression pattern showed a significant increase in the serum concentration immediately after surgery, which might contribute to a steeper increase in the VEGF concentration due to a costimulatory effect.

We could show that the local application of BMP-7 leads to a modulation of the VEGF expression pattern by a complex costimulatory response. In this clinical observer study, we were able to confirm results solely gathered in a previously published in vitro study. ${ }^{38}$ Furthermore, our data indicate that the biological impact of BMP-7 leads to an increased and earlier VEGF expression pattern by an additional indirect 
stimulation of VEGF expression by a timely stimulation of the IL-6, IL-8, and TNF- $\alpha$ expressions.

\section{Inflammation}

Physiological fracture healing requires a sufficient angiogenesis as well as an adequate stimulus to trigger the beginning of bone healing. Multiple studies have shown that an early inflammatory response serves as a stimulus for bone healing. The inflammatory response is characterized by several cytokines. The proinflammatory cytokines IL-6, TNF- $\alpha$, and IL- 8 are expressed as important initiators of the acute-phase response. ${ }^{44}$ An increase in the serum values can be observed shortly after fracture. ${ }^{12,45}$ It is known that the local application of BMP-7 may lead to local signs of inflammation, such as soft-tissue swelling, edema, and reddening of the skin. Although this BMP-mediated soft-tissue swelling is not fully understood, it has been associated with recent studies on the increased incident of proinflammatory cytokines (IL-6, IL-8, and TNF- $\alpha$ ). ${ }^{46-48} \mathrm{~A}$ recent study found that the application of BMP-7 modulates the microenvironment at the fracture side, in particular by altering the cytokine expression, and thereby, osteoclastogenesis is inhibited. ${ }^{47}$ Furthermore, as confirmed by our study, no signs of systemic inflammation were detected (both clinically and assessed by the comparison of CRP and leukocyte serum levels in-between groups). Triggered by these cytokines, inflammatory cells such as leukocytes and macrophages migrate into the hematoma caused by injuries of vessels by the fracture. ${ }^{49,50}$ The interfragmentary hematoma establishes a cellular foundation for the following bone healing. ${ }^{18,51}$ During this initial inflammatory response, various proinflammatory signals and growth factors are released. Among these proinflammatory cytokines, IL-6 and TNF- $\alpha$ play an important role.

In particular, the inflammatory mediator IL-6 has been shown to be upregulated in early fracture healing. ${ }^{52,53}$ IL-6 causes mesenchymal stem cells to differentiate to osteoblasts, ${ }^{54,55}$ thereby promoting bone regeneration. A delayed callus formation, mineralization, and remodeling were found in IL-6 knockout mice. ${ }^{56}$ Additionally, IL-6 is responsible for the immigration of leukocytes into the fracture site. ${ }^{49}$ Significant differences in the IL-6 serum levels between the study group and control group were detected after 2 days and 6 weeks. The BMP-7 group showed an increase in the peak serum levels immediately after the local application of BMP-7, whereas the peak serum levels in the control group were significantly lower. Especially, the stimulation of the expression of IL-6 on day 2 is relevant as it represents the initial inflammatory response. BMP-7 is therefore capable of inducing the expression of IL-6, which may induce an increased inflammatory response. In conclusion, application of BMP-7 leads to a significantly higher expression of IL-6 during the initial inflammatory response.

TNF- $\alpha$ is released by macrophages and inflammatory cells. ${ }^{20}$ The initial release of TNF- $\alpha$ stimulates further expression of several inflammatory mediators and causes immigration of cells into the fracture site. ${ }^{45}$ According to demand, TNF- $\alpha$ triggers apoptosis or secures the survival of cells. ${ }^{57}$ The TNF- $\alpha$-associated receptors are TNFR-1 and TNFR-2, which are located on cellular surfaces. ${ }^{45}$ While TNFR-1 is permanently present, TNFR-2 is only expressed after fracture. TNF- $\alpha$ stimulation via TNFR-1 causes the differentiation of osteoclasts and therefore stimulates bone resorption. TNF- $\alpha$ stimulation via TNFR-2 leads to a contrary reaction and is a stimulus for bone regeneration..$^{20,58}$ Our data showed an earlier and higher expression of TNF- $\alpha$ during the initial phase of bone healing after the local application of BMP-7. This may contribute to an earlier and higher influence on TNFR-2, resulting in favorable bone regeneration. However, the differences in the TNF- $\alpha$ expression level between both groups were at a nonsignificant extend; therefore, further studies are needed to prove this postulation.

IL-8 induces an acute inflammatory response via activation and immigration of neutrophile granulocytes. ${ }^{59}$ Fuller et $a l^{60}$ reported that expression of IL- 8 inhibits bone resorption in isolated rat osteoclasts by a decreased proportion of osteoclasts resorbing bone. A recent study showed that IL-8 is important for the regulation of bone resorption by direct stimulation of IL-8 on osteoclastogenesis. ${ }^{61}$ IL-8 expression depends on a variety of factors, such as the presence and effects of TNF- $\alpha$ and IL- $6 .{ }^{62}$ The results of our study show similar expression pattern of both TNF- $\alpha$ and IL-8. The control group showed an IL-8 peak after 4 weeks of surgery, while IL- 8 levels in the BMP-7 group peaked after 2 weeks. Thereby, the results of our study indicate a complex interaction between multiple cytokines subsequent to the application of BMP-7. Despite existing studies, the impact of IL-8 on bone regeneration at the fracture site is not yet fully understood. ${ }^{60-62}$ In our study, the increase in the IL-8 expression during the initial inflammatory response subsequent to the application of BMP-7 indicates the role of IL-8 as a proinflammatory cytokine in bone regeneration. However, further studies are needed to prove this contribution to bone healing.

\section{Limitations}

Nonunions are a severe but an infrequent complication; this is why, clinical studies concerning nonunion therapy are 
complex and it explains the small size of our patient collective. Despite the large patient collective, just a small number of patients could be included in the study due to our strict inclusion and exclusion criteria. In the context of the current literature and our recent studies, ${ }^{22,23}$ the collective size of our patients is still sufficient. Another limiting factor is the treatment of two patients with plate fixation and eight patients with nail fixation in the BMP-7 group, whereas patients in the control group received plate fixation in nine cases and nail fixation in one case. The reason for different fixation methods in our study groups is the collaboration between two clinical centers. A comparison of both therapy methods characterized an equal inflammatory response after plate fixation or intramedullar nailing as a treatment for tibial fractures. Furthermore, the responder rate, the development of nonunions, and the number of following surgeries were similar. ${ }^{63}$ The results of our study may be influenced by a systemic inflammation; therefore, we assessed the CRP and leukocyte serum patterns in the initial 2 weeks subsequent to the procedure. Our data show that the CRP values, after an expected peak after 2 days of surgery, decreased in both groups; furthermore, the CRP values in the control group were even higher than in the study group. Leukocytes stayed in a physiological range during the whole time, thereby indicating that no systemic inflammation was present and reducing the risk of influences on our study results. The preoperative serum levels of VEGF showed differences between both groups. This might influence the analysis of the BMP-7 effects. However, in our statistical analysis, we could see a steeper and faster increase in the VEGF serum levels. Furthermore, despite an overall similarVEGF expression pattern in both groups, VEGF levels rose higher and faster in the BMP-7 group. Therefore, the differences in the baseline do not interfere with the scientific results of our study. Another limitation of our study is a missing fracture group, in which cytokine expression patterns of physiological bone healing are analyzed. However, it is known that the microbiology in patients with delayed and failed bone healing is different. Therefore, we believe that the missing fracture group is not interfering with the results of our study.

\section{Conclusion}

The patients with failed fracture healing are known to have diminished concentrations of BMP. The local application of BMP served as substitution, thereby modulating the microenvironment in the fracture gap and increasing local concentrations of BMP-7. In this study, we were able to analyze the influence of BMP-7 application on the expression pattern of inflammable and angiogenic cytokines. Up-to-date multiple studies utilized animal models in order to assess the biological impact of BMP-7, leaving the bias of differences in the reaction of the immune system. Therefore, quantitative measurement of human serum cytokine expression provides a valid instrument in evaluating the biological impact of BMP-7.7,23,64

The local application of BMP-7 in addition to autologous bone graft transplantation provides better bone healing. ${ }^{13,14}$ Our findings state that BMP-7 via a direct and a postulated indirect pathway by costimulatory effects on proinflammatory cytokine expression leads to an increased VEGF expression. Moreover, the application of BMP-7 leads to an earlier and higher expression of inflammable cytokines; thereby, the application of BMP-7 may contribute to an inflammatory response functioning as a possible stimulus for bone regeneration. Significant higher IL-6 levels and an earlier peak of IL-6, IL-8, and TNF- $\alpha$ were observed in the BMP-7 group. In conclusion, the application of BMP-7 modulates both angiogenic and inflammable cytokine expression patterns.

\section{Acknowledgment}

We would like to thank Martina Kutsche-Bauer for performing the Luminex assays.

\section{Disclosure}

The authors report no conflicts of interest in this work.

\section{References}

1. Giannoudis PV, Einhorn TA, Marsh D. Fracture healing: the diamond concept. Injury. 2007;38(suppl 4):S3-S6.

2. Takemoto R, Forman J, Taormina DP, Egol KA. No advantage to rhBMP-2 in addition to autogenous graft for fracture nonunion. Orthopedics. 2014;37(6): e525-e530.

3. Schmidmaier G, Schwabe P, Wildemann B, Haas NP. Use of bone morphogenetic proteins for treatment of non-unions and future perspectives. Injury. 2007;38(suppl 4):S35-S41.

4. Giannoudis PV, Einhorn TA, Schmidmaier G, Marsh D. The diamond concept - open questions. Injury. 2008;39(suppl 2):S5-S8.

5. Zimmermann G, Moghaddam A, Reumann M, et al. TGF- $\beta 1$ als pathophysiologischer Faktor bei der Frakturheilung [TGF-beta1 as a pathophysiological factor in fracture healing]. Unfallchirurg. 2007;110(2):130-136. German.

6. Donegan DJ, Scolaro J, Matuszewski PE, Mehta S. Staged bone grafting following placement of an antibiotic spacer block for the management of segmental long bone defects. Orthopedics. 2011;34(11):e730-e735.

7. Moghaddam A, Zietzschmann S, Bruckner T, Schmidmaier G. Treatment of atrophic tibia non-unions according to 'diamond concept': results of one- and two-step treatment. Injury. 2015;46(suppl 4):S39-S50.

8. Schmidmaier G, Moghaddam A. Pseudarthrosen langer Röhrenknochen [Long Bone Nonunion]. Z Orthop Unfall. 2015;153(6):659-676. German

9. Christou C, Oliver RA, Yu Y, Walsh WR. The Masquelet technique for membrane induction and the healing of ovine critical sized segmental defects. PLoS One. 2014;9(12):e114122.

10. Gautschi OP, Frey SP, Zellweger R. Bone morphogenetic proteins in clinical applications. ANZ J Surg. 2007;77(8):626-631.

11. Borrelli J Jr, Prickett WD, Ricci WM. Treatment of nonunions and osseous defects with bone graft and calcium sulfate. Clin Orthop Relat Res. 2003;411:245-254. 
12. Cho TJ, Gerstenfeld LC, Einhorn TA. Differential temporal expression of members of the transforming growth factor beta superfamily during murine fracture healing. J Bone Miner Res. 2002;17(3):513-520.

13. Giannoudis PV, Kanakaris NK, Dimitriou R, Gill I, Kolimarala V, Montgomery RJ. The synergistic effect of autograft and BMP-7 in the treatment of atrophic nonunions. Clin Orthop Relat Res. 2009;467(12):3239-3248

14. Moghaddam-Alvandi A, Zimmermann G, Buchler A, et al. Ergebnisse der Pseudarthrosenbehandlung mit "bone morphogenetic protein 7" (BMP-7) [Results of nonunion treatment with bone morphogenetic protein 7 (BMP-7)]. Unfallchirurg. 2012;115(6):518-526. German.

15. Dai J, Li L, Jiang C, Wang C, Chen H, Chai Y. Bone morphogenetic protein for the healing of tibial fracture: a meta-analysis of randomized controlled trials. PLoS One. 2015;10(10):e0141670.

16. Lee KB, Taghavi CE, Murray SS, Song KJ, Keorochana G, Wang JC. BMP induced inflammation: a comparison of rhBMP-7 and rhBMP-2. J Orthop Res. 2012;30(12):1985-1994.

17. Phillips AM. Overview of the fracture healing cascade. Injury. 2005;36(suppl 3):S5-S7.

18. Pape HC, Marcucio R, Humphrey C, Colnot C, Knobe M, Harvey EJ. Trauma-induced inflammation and fracture healing. J Orthop Trauma. 2010;24(9):522-525.

19. Croes M, Oner FC, Kruyt MC, et al. Proinflammatory mediators enhance the osteogenesis of human mesenchymal stem cells after lineage commitment. PLoS One. 2015;10(7):e0132781.

20. Kon T, Cho TJ, Aizawa T, et al. Expression of osteoprotegerin, receptor activator of NF-kappaB ligand (osteoprotegerin ligand) and related proinflammatory cytokines during fracture healing. J Bone Miner Res. 2001;16(6):1004-1014.

21. Street J, Bao M, deGuzman L, et al. Vascular endothelial growth factor stimulates bone repair by promoting angiogenesis and bone turnover. Proc Natl Acad Sci U S A. 2002;99(15):9656-9661.

22. Moghaddam A, Muller U, Roth HJ, Wentzensen A, Grutzner PA, Zimmermann G. TRACP $5 \mathrm{~b}$ and CTX as osteological markers of delayed fracture healing. Injury. 2011;42(8):758-764.

23. Westhauser F, Zimmermann G, Moghaddam S, et al. Reaming in treatment of non-unions in long bones: cytokine expression course as a tool for evaluation of non-union therapy. Arch Orthop Trauma Surg. 2015;135(8):1107-1116.

24. Bender D, Haubruck P, Boxriker S, Korff S, Schmidmaier G, Moghaddam A. Validity of subjective smoking status in orthopedic patients. Ther Clin Risk Manag. 2015;11:1297-1303.

25. Zimmermann G, Wagner C, Schmeckenbecher K, Wentzensen A, Moghaddam A. Treatment of tibial shaft non-unions: bone morphogenetic proteins versus autologous bone graft. Injury. 2009;40(suppl 3):S50-S53.

26. Kanczler JM, Oreffo RO. Osteogenesis and angiogenesis: the potential for engineering bone. Eur Cell Mater. 2008;15:100-114.

27. Lu C, Marcucio R, Miclau T. Assessing angiogenesis during fracture healing. Iowa Orthop J. 2006;26:17-26.

28. Hausman MR, Schaffler MB, Majeska RJ. Prevention of fracture healing in rats by an inhibitor of angiogenesis. Bone. 2001;29(6):560-564.

29. Street J, Bao M, deGuzman L, et al. Vascular endothelial growth factor stimulates bone repair by promoting angiogenesis and bone turnover. Proc Natl Acad Sci U S A. 2002;99(15):9656-9661.

30. Beamer B, Hettrich C, Lane J. Vascular endothelial growth factor: an essential component of angiogenesis and fracture healing. HSS J. 2010;6(1):85-94.

31. Sarahrudi K, Thomas A, Braunsteiner T, Wolf H, Vecsei V, Aharinejad S. VEGF serum concentrations in patients with long bone fractures: a comparison between impaired and normal fracture healing. J Orthop Res. 2009;27(10):1293-1297.

32. Young S, Patel ZS, Kretlow JD, et al. Dose effect of dual delivery of vascular endothelial growth factor and bone morphogenetic protein-2 on bone regeneration in a rat critical-size defect model. Tissue Eng Part A. 2009;15(9):2347-2362.

33. Ogilvie CM, Lu C, Marcucio R, et al. Vascular endothelial growth factor improves bone repair in a murine nonunion model. Iowa Orthop J. 2012;32:90-94.
34. Eckardt H, Ding M, Lind M, Hansen ES, Christensen KS, Hvid I. Recombinant human vascular endothelial growth factor enhances bone healing in an experimental nonunion model. J Bone Joint Surg Br. 2005;87(10):1434-1438.

35. Tarkka T, Sipola A, Jamsa T, et al. Adenoviral VEGF-A gene transfer induces angiogenesis and promotes bone formation in healing osseous tissues. J Gene Med. 2003;5(7):560-566.

36. Ramoshebi LN, Ripamonti U. Osteogenic protein-1, a bone morphogenetic protein, induces angiogenesis in the chick chorioallantoic membrane and synergizes with basic fibroblast growth factor and transforming growth factor-beta1. Anat Rec. 2000;259(1):97-107.

37. Langenfeld EM, Langenfeld J. Bone morphogenetic protein-2 stimulates angiogenesis in developing tumors. Mol Cancer Res. 2004; 2(3):141-149.

38. Suzuki Y, Montagne K, Nishihara A, Watabe T, Miyazono K. BMPs promote proliferation and migration of endothelial cells via stimulation of VEGF-A/VEGFR2 and angiopoietin-1/Tie2 signalling. J Biochem. 2008; 143(2):199-206.

39. Akiyama I, Yoshino O, Osuga Y, et al. Bone morphogenetic protein 7 increased vascular endothelial growth factor (VEGF)-a expression in human granulosa cells and VEGF receptor expression in endothelial cells. Reprod Sci. 2014;21(4):477-482.

40. Rega G, Kaun C, Demyanets S, et al. Vascular endothelial growth factor is induced by the inflammatory cytokines interleukin- 6 and oncostatin $\mathrm{m}$ in human adipose tissue in vitro and in murine adipose tissue in vivo. Arterioscler Thromb Vasc Biol. 2007;27(7):1587-1595.

41. Cohen T, Nahari D, Cerem LW, Neufeld G, Levi BZ. Interleukin 6 induces the expression of vascular endothelial growth factor. $J$ Biol Chem. 1996;271(2):736-741.

42. Heidemann J, Ogawa H, Dwinell MB, et al. Angiogenic effects of interleukin 8 (CXCL8) in human intestinal microvascular endothelial cells are mediated by CXCR2. J Biol Chem. 2003;278(10): 8508-8515.

43. Johnson KE, Wilgus TA. Vascular endothelial growth factor and angiogenesis in the regulation of cutaneous wound repair. Adv Wound Care (New Rochelle). 2014;3(10):647-661.

44. Gruys E, Toussaint MJM, Niewold TA, Koopmans SJ. Acute phase reaction and acute phase proteins. J Zhejiang Univ Sci B. 2005;6(11):1045-1056.

45. Mountziaris PM, Mikos AG. Modulation of the inflammatory response for enhanced bone tissue regeneration. Tissue Eng Part B Rev. 2008;14(2): 179-186.

46. Lee KB, Taghavi CE, Song KJ, et al. Inflammatory characteristics of rhBMP-2 in vitro and in an in vivo rodent model. Spine. 2011;36(3): E149-E154.

47. Maurer T, Zimmermann G, Maurer S, Stegmaier S, Wagner C, Hansch GM. Inhibition of osteoclast generation: a novel function of the bone morphogenetic protein 7/osteogenic protein 1. Mediators Inflamm. 2012;2012:171209.

48. Robin BN, Chaput CD, Zeitouni S, Rahm MD, Zerris VA, Sampson HW. Cytokine-mediated inflammatory reaction following posterior cervical decompression and fusion associated with recombinant human bone morphogenetic protein-2: a case study. Spine. 2010;35(23):E1350-E1354.

49. Bastian O, Pillay J, Alblas J, Leenen L, Koenderman L, Blokhuis T. Systemic inflammation and fracture healing. J Leukoc Biol. 2011;89(5):669-673.

50. Campbell DJ, Kim CH, Butcher EC. Chemokines in the systemic organization of immunity. Immunol Rev. 2003;195:58-71.

51. Kolar P, Gaber T, Perka C, Duda GN, Buttgereit F. Human early fracture hematoma is characterized by inflammation and hypoxia. Clin Orthop Relat Res. 2011;469(11):3118-3126.

52. Rundle $\mathrm{CH}$, Wang $\mathrm{H}, \mathrm{Yu} \mathrm{H}$, et al. Microarray analysis of gene expression during the inflammation and endochondral bone formation stages of rat femur fracture repair. Bone. 2006;38(4):521-529.

53. Gerstenfeld LC, Cullinane DM, Barnes GL, Graves DT, Einhorn TA. Fracture healing as a post-natal developmental process: molecular, spatial, and temporal aspects of its regulation. $J$ Cell Biochem. 2003;88(5):873-884. 
54. Kudo O, Sabokbar A, Pocock A, Itonaga I, Fujikawa Y, Athanasou NA. Interleukin-6 and interleukin-11 support human osteoclast formation by a RANKL-independent mechanism. Bone. 2003;32(1):1-7.

55. Heymann D, Rousselle AV. gp130 Cytokine family and bone cells. Cytokine. 2000;12(10):1455-1468.

56. Yang X, Ricciardi BF, Hernandez-Soria A, Shi Y, Pleshko Camacho N, Bostrom MP. Callus mineralization and maturation are delayed during fracture healing in interleukin-6 knockout mice. Bone. 2007;41(6):928-936

57. Locksley RM, Killeen N, Lenardo MJ. The TNF and TNF receptor superfamilies: integrating mammalian biology. Cell. 2001;104(4):487-501.

58. Balga R, Wetterwald A, Portenier J, Dolder S, Mueller C, Hofstetter W. Tumor necrosis factor-alpha: alternative role as an inhibitor of osteoclast formation in vitro. Bone. 2006;39(2):325-335.

59. Harada A, Sekido N, Akahoshi T, Wada T, Mukaida N, Matsushima K. Essential involvement of interleukin-8 (IL-8) in acute inflammation. $J$ Leukoc Biol. 1994;56(5):559-564.
60. Fuller K, Owens JM, Chambers TJ. Macrophage inflammatory protein-1 alpha and IL-8 stimulate the motility but suppress the resorption of isolated rat osteoclasts. J Immunol. 1995;154(11):6065-6072.

61. Bendre MS, Montague DC, Peery T, Akel NS, Gaddy D, Suva LJ. Interleukin- 8 stimulation of osteoclastogenesis and bone resorption is a mechanism for the increased osteolysis of metastatic bone disease. Bone. 2003;33(1):28-37.

62. DeForge LE, Preston AM, Takeuchi E, Kenney J, Boxer LA, Remick DG. Regulation of interleukin 8 gene expression by oxidant stress. $J$ Biol Chem. 1993;268(34):25568-25576.

63. Vallier HA, Cureton BA, Patterson BM. Randomized, prospective comparison of plate versus intramedullary nail fixation for distal tibia shaft fractures. J Orthop Trauma. 2011;25(12):736-741.

64. Moghaddam A, Child C, Bruckner T, Gerner HJ, Daniel V, Biglari B. Posttraumatic inflammation as a key to neuroregeneration after traumatic spinal cord injury. Int J Mol Sci. 2015;16(4):7900-7916.
Journal of Inflammation Research

\section{Publish your work in this journal}

The Journal of Inflammation Research is an international, peer-reviewed open access journal that welcomes laboratory and clinical findings on the molecular basis, cell biology and pharmacology of inflammation including original research, reviews, symposium reports, hypothesis formation and commentaries on: acute/chronic inflammation; mediators of

\section{Dovepress}

inflammation; cellular processes; molecular mechanisms; pharmacology and novel anti-inflammatory drugs; clinical conditions involving inflammation. The manuscript management system is completely online and includes a very quick and fair peer-review system. Visit http://www.dove press.com/testimonials.php to read real quotes from published authors.

Submit your manuscript here: https://www.dovepress.com/journal-of-inflammation-research-journal 